Portland State University

PDXScholar

3-1-2003

\title{
High-resolution near-field Raman microscopy of single-walled carbon nanotubes
}

Achim Hartschuh

X. Sunney Xie

Lukas Novotny

Erik J. Sánchez

Follow this and additional works at: https://pdxscholar.library.pdx.edu/phy_fac

Part of the Physics Commons

Let us know how access to this document benefits you.

\section{Citation Details}

Hartschuh, A., Sánchez, E., Xie, X., \& Novotny, L. (2003). High-resolution near-field Raman microscopy of single-walled carbon nanotubes. Physical Review Letters, 90(9), 095503.

This Article is brought to you for free and open access. It has been accepted for inclusion in Physics Faculty Publications and Presentations by an authorized administrator of PDXScholar. Please contact us if we can make this document more accessible: pdxscholar@pdx.edu. 


\title{
High-Resolution Near-Field Raman Microscopy of Single-Walled Carbon Nanotubes
}

\author{
Achim Hartschuh, ${ }^{1}$ Erik J. Sánchez, ${ }^{2}$ X. Sunney Xie, ${ }^{3}$ and Lukas Novotny ${ }^{1}$ \\ ${ }^{1}$ The Institute of Optics, University of Rochester, Rochester, New York 14627 \\ ${ }^{2}$ Department of Physics, Portland State University, Portland, Oregon 97207 \\ ${ }^{3}$ Department of Chemistry and Chemical Biology, Harvard University, Cambridge, Massachusetts 02138
}

(Received 9 August 2002; published 4 March 2003)

\begin{abstract}
We present near-field Raman spectroscopy and imaging of single isolated single-walled carbon nanotubes with a spatial resolution of $\approx 25 \mathrm{~nm}$. The near-field origin of the image contrast is confirmed by the measured dependence of the Raman scattering signal on tip-sample distance and the unique polarization properties. The method is used to study local variations in the Raman spectrum along a single single-walled carbon nanotube.
\end{abstract}

DOI: 10.1103/PhysRevLett.90.095503

Recent rapid advances in nanotechnology and nanoscience are largely due to our newly acquired ability to measure and manipulate individual structures on the nanoscale. Among the new methods are scanning probe techniques, optical tweezers, and high-resolution electron microscopes. Recently, a near-field optical technique has been demonstrated which allows spectroscopic measurements with $20 \mathrm{~nm}$ spatial resolution [1]. The method makes use of the strongly enhanced electric field close to a sharp metal tip under laser illumination and relies on the detection of two-photon excited fluorescence. However, fluorescence imaging requires a high fluorescence quantum yield of the system studied or artificial labeling with fluorophores. Furthermore, fluorescence quenching by the metal tip competes with the local field enhancement effect and therefore limits the general applicability. On the other hand, Raman scattering probes the unique vibrational spectrum of the sample and directly reflects its chemical composition and molecular structure. A main drawback of Raman scattering is the extremely low scattering cross section which is typically 14 orders of magnitude smaller than the cross section of fluorescence. Surface enhanced Raman scattering (SERS), induced by nanometer-sized metal structures, has been shown to provide enormous enhancement factors of up to $10^{15}$ allowing for Raman spectroscopy even on the single molecule level $[2,3]$. Controlling SERS with a sharp metal tip which is raster scanned over a sample surface has been proposed [1,4], and near-field Raman enhancement has been experimentally demonstrated [5-9]. Here, we show the chemical specificity of this near-field technique and demonstrate an unprecedented spatial resolution.

Single-walled carbon nanotubes (SWNTs) have been the focus of intense interest due to a large variety of potential nanotechnological applications. The unique properties of SWNTs arise from their particular onedimensional structure which is directly linked to the characteristic Raman bands. Raman scattering on SWNTs has been studied intensively in the literature
PACS numbers: 61.46.+w, 07.79.-v, 78.30.Na, 78.67.Ch

(see, e.g., Refs. [10-13]) and Raman enhancements of up to $10^{12}$ have been reported for tubes in contact with fractal silver colloidal clusters [14]. In this Letter, nearfield Raman imaging of SWNTs is demonstrated using a sharp silver tip as a probe. We show, for the first time, that single isolated SWNTs can be detected optically with a spatial resolution better than $30 \mathrm{~nm}$. This high-resolution capability is applied to resolve local variations in the Raman spectrum along a single SWNT which would be hidden in far-field measurements. The near-field origin of the Raman enhancement is proven by tip-sample distance measurements.

Our experimental setup is based on an inverted optical microscope with an $x, y$ scan stage for raster scanning a transparent sample with SWNTs. A laser beam $(\lambda=$ $633 \mathrm{~nm}, 30-100 \mu \mathrm{W}$ ) is reflected by a dichroic beam splitter and focused by a high numerical aperture (NA) objective (1.4NA) on the sample surface. A sharp silver tip is positioned near the focus of the beam and maintained above the sample surface at a distance of $\approx 1 \mathrm{~nm}$ by means of a sensitive shear-force feedback mechanism [15]. Raman scattered light is collected with the same objective, transmitted by the beam splitter and filtered by a long pass filter. The signal is detected either by a combination of a spectrograph and a thermoelectrically cooled charged coupled device or by a narrow band pass filter $(\mathrm{FWHM}=10 \mathrm{~nm})$ centered at $760 \mathrm{~nm}($ or $700 \mathrm{~nm})$ followed by a single-photon counting avalanche photodiode. A near-field Raman image is established by raster scanning the sample and continuously recording the scattered Raman signal. Sharp silver tips with a radius of $10-15 \mathrm{~nm}$ are produced by electrochemical etching followed by focused ion beam (FIB) milling using a dual beam FIB system (FEI Strata-235) and utilizing a similar procedure as used for STM/AFM (scanning tunneling microscopy/atomic force microscopy) tip sharpening [16].

To establish a strong field enhancement at the tip, the electric field of the exciting laser beam needs to be polarized along the tip axis [17]. To achieve this condition in our on-axis illumination scheme, we displace the tip 
from the center of the beam in the direction of polarization into one of the two longitudinal field lobes characteristic for strongly focused Gaussian beams [18]. The strength of this longitudinal field increases with increasing NA of the focusing lens, and in our configuration, is only 5 times weaker than the transverse field strength at the center of the focus.

Two different types of SWNT samples were studied. The first sample consists of nearly monodisperse SWNTs grown by chemical vapor deposition (CVD) on $\mathrm{SiO}_{2}$ substrates. The substrates were first cleaned by solvent washing then heated to $700{ }^{\circ} \mathrm{C}$ in a tube furnace for 30 min while being exposed to air. $50 \mu \mathrm{l}$ of Ferritin solution [Sigma-Aldrich, diluted in deionized (DI) water, 1:10 ratio (Ferritin: $\mathrm{H}_{2} \mathrm{O}$ )] was applied to the clean substrate which was left for $30 \mathrm{~s}$ then rinsed with DI water, then placed in the tube furnace using growth parameters similar to commonly known SWNT growth methods [19]. The material for the second sample was purchased from BuckyUSA and consists of purified SWNTs with a diameter distribution from 1.0-1.8 $\mathrm{nm}$, produced by arc discharge using $\mathrm{Ni} / \mathrm{Y}$ catalyst particles. The material was dispersed in dichlorethane, sonicated for $1 \mathrm{~h}$ and spin cast on a microscope cover glass. AFM measurements were performed to determine sample coverage and to distinguish spatially isolated tubes from nanotube bundles.

The far-field Raman spectra of the SWNT samples (not shown) are dominated by the sharp tangential stretching mode at $1596 \mathrm{~cm}^{-1}$ ( $G$ band) and a broader band around $2615 \mathrm{~cm}^{-1}\left(G^{\prime}\right.$ band) which arises from an overtone of the disorder induced mode around $1310 \mathrm{~cm}^{-1}$ [20]. Figure 1 shows confocal far-field Raman images of SWNT bundles produced by arc discharge which were recorded by integrating the signal intensity of the $G^{\prime}$ band (760 $\mathrm{nm}$ band pass filter, see above) for vertical (a) and horizontal (b) polarization of the incident laser beam.
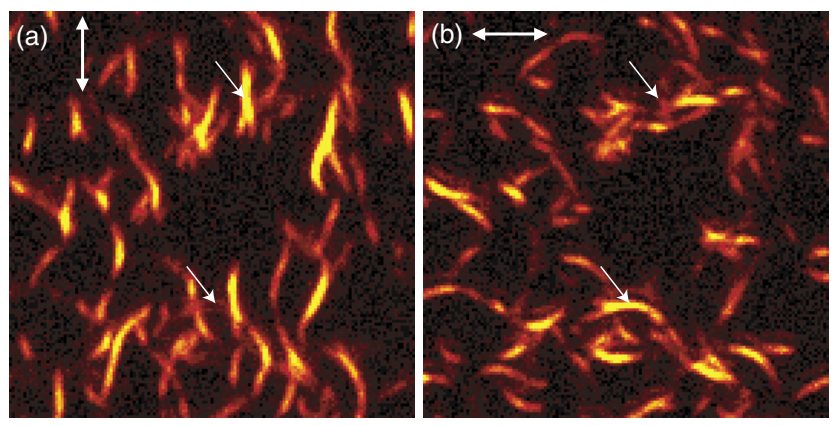

FIG. 1 (color online). Confocal Raman images of SWNT bundles produced by arc discharge on glass acquired by detecting the intensity of the $G^{\prime}$ band $\left(\nu=2615 \mathrm{~cm}^{-1}\right)$ while raster scanning the sample (without metal tip, scan area $15 \times 15 \mu \mathrm{m}$ ) for vertical (a) and horizontal polarization (b) of the laser excitation at $633 \mathrm{~nm}$. The arrows indicate tubes which are oriented completely perpendicular to one polarization direction demonstrating the polarization contrast.
Similar Raman images are obtained by detecting the intensity of the $G$ band using the $700 \mathrm{~nm}$ band pass filter. The Raman images clearly demonstrate that tubes oriented completely perpendicular to the respective light polarization do not produce any signal (see arrows in Fig. 1). This polarization contrast is a consequence of the polarization properties of the $G$ and $G^{\prime}$ Raman bands reported for $G$ in the literature [11,12,21]. AFM measurements of the same area reveal that the diameter of the nanotube bundles varies between 5-15 $\mathrm{nm}$ which corresponds to $10-100$ individual nanotubes per bundle.

Confocal Raman imaging of SWNTs grown by CVD was not possible within feasible acquisition times because the sample consists mostly of separated individual SWNTs for which the Raman scattering strength is much weaker. However, as shown in Fig. 2(a) for CVD grown SWNTs, the contrast and resolution of Raman images can be greatly increased by positioning a sharp silver tip near the laser focus. The simultaneously recorded topographic image is presented in Fig. 2(b). The lower portions [Figs. 2(c) and 2(d)] show cross sections taken along the dashed white lines. Besides SWNTs, the topographic image shows a large number of small circular features with a height of $\approx 2 \mathrm{~nm}$ which are due to condensating water and can be removed by sample heating to $70{ }^{\circ} \mathrm{C}$. Comparison of the widths (FWHM) of SWNTs

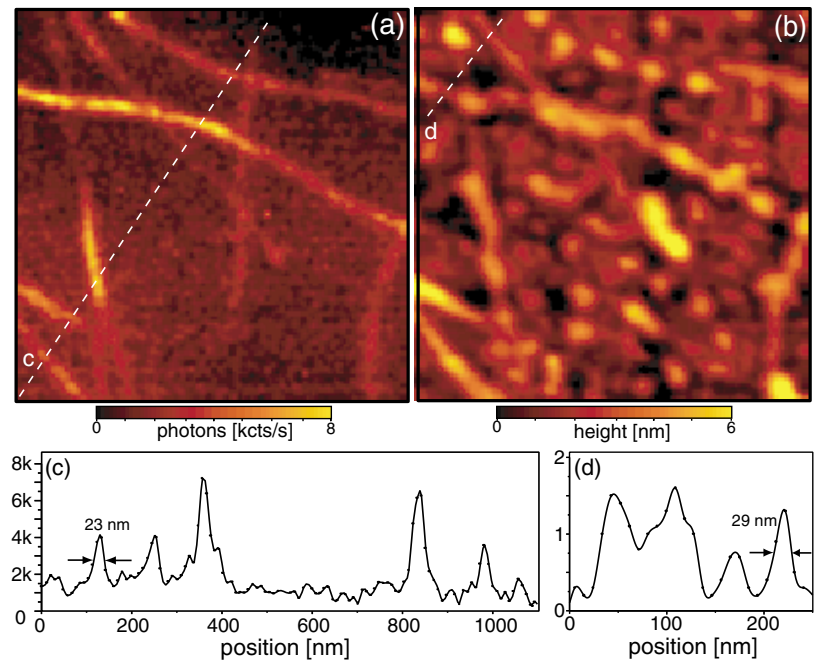

FIG. 2 (color online). Simultaneous near-field Raman image (a) and topographic image (b) of SWNTs grown by CVD on glass. Scan area $1 \times 1 \mu \mathrm{m}$. The Raman image is acquired by detecting the intensity of the $G^{\prime}$ band upon laser excitation at $633 \mathrm{~nm}$. No Raman scattering signal is detected from humidity related circular features present in the topographic image (see text). (c) Cross section taken along the indicated dashed line in the Raman image. (d) Cross section taken along the indicated dashed line in the topographic image. The height of individual tubes is $\approx 1.4 \mathrm{~nm}$. Vertical units are photon counts per second for (c) and nanometer for (d). 
in the topographic and Raman image indicate that the resolution of the Raman image is better than the resolution of the topographic image ( $25 \mathrm{vs} 30 \mathrm{~nm}$ ). This high spatial resolution is unprecedented and clearly indicates the near-field origin of the Raman signal. The widths of the optical features and the topographical features result from the convolution of the tip shape with the object studied and are therefore limited by the finite size of the tip apex. Both images are closely correlated and the SWNTs are clearly identified. However, the small topographic features formed by condensation do not show up in the Raman image which proves the chemical specificity of the method. The diameter of SWNTs grown by CVD can be controlled by growth parameters. The sample used in Fig. 2 consists mostly of SWNTs with a diameter of $\approx 1.4 \mathrm{~nm}$ which is verified by the topographic data in Figs. 2(b) and 2(d) and also by independent AFM measurements in the absence of water condensation. Thus, we observe near-field Raman scattering from spatially separated single SWNTs. It is important to notice that vertically and horizontally oriented SWNTs are observed in the Raman image with similar signal intensity even though the incident laser light is oriented vertically. This observation is in strong contrast to the polarization contrast observed in the far-field measurements shown in Fig. 1. Accordingly, the polarization of the enhanced field has to be radially symmetric with respect to the tip axis. This finding is in agreement with theoretical calculations [17] and confirms the unique properties of the near field.

The optical resolution apparent in Fig. 2 shows that the enhanced field is laterally confined to the size of the metal tip. To demonstrate the confinement of the enhanced fields in longitudinal direction, the tip is positioned above a SWNT and the Raman scattering strength is recorded as a function of tip-sample distance $\Delta z$. The result, shown in Fig. 3, has been fitted with an exponential curve and normalized with the Raman scattering strength in the absence of the tip, i.e., with the far-field Raman signal. For distances $\Delta z<30 \mathrm{~nm}$ the increase of the signal clearly indicates a distance dependent near-field contribution with a decay length of $11 \mathrm{~nm}$. This longitudinal confinement is in agreement with the lateral confinement observed in the near-field Raman images and consistent with the tip radius of $10-15 \mathrm{~nm}$ as determined by scanning electron microscopy (SEM) [c.f. Fig. 3(b)]. All the tips used in our experiments were fabricated by the same procedure and rendered similar SEM images. However, only about $30 \%$ to $40 \%$ of the tips produced a measurable signal enhancement. The reason for this behavior is the subject of ongoing studies. The highest Raman enhancement factors observed so far were estimated to be around $10^{3}$. These numbers follow from the detected ratio of near-field to far-field signals and from a comparison of the different volumes probed by the near field and the far field.

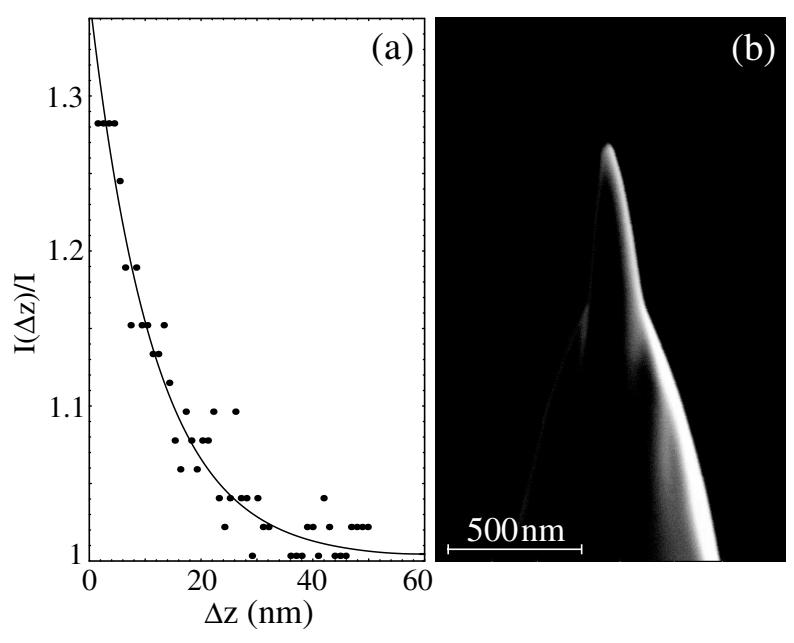

FIG. 3. (a) Dependence of the Raman scattering strength of the $G^{\prime}$ band $(I)$ on the longitudinal separation $(\Delta z)$ between a single SWNT and the tip. The solid line is an exponential fit with a decay length of $11 \mathrm{~nm}$. The signal is normalized with the far-field signal. (b) Scanning electron micrograph of a sharp silver tip fabricated by focused ion beam milling.

To demonstrate the high-resolution spectroscopic capabilities of the here presented near-field technique we investigate the Raman spectrum along a SWNT with a diameter of $1.7 \mathrm{~nm}$ grown by arc discharge. Figure 4(a) shows the topography near the end of a SWNT produced by this technique. Three bumps with a height of $\approx 5 \mathrm{~nm}$ can be recognized on top of the SWNT. Because of their size, these bumps are presumably $\mathrm{Ni} / \mathrm{Y}$ catalyst particles indicating the initial point of growth [22]. The labels 1 to 4 in Fig. 4(a) mark the positions at which Raman spectra were taken in the presence of the metal tip. At the very beginning of the SWNT (positions 1 and 2 ) the $G$ band at $1596 \mathrm{~cm}^{-1}\left(\mathrm{FWHM}=17 \mathrm{~cm}^{-1}\right)$ dominates the spectra and is significantly stronger than the $G^{\prime}$ band at $2619 \mathrm{~cm}^{-1}$ (FWHM $=35 \mathrm{~cm}^{-1}$ ) with an amplitude ratio $G / G^{\prime}$ of $\approx 1.3$ [see Fig. 4(b)]. However, moving along the SWNT to position 3 and further to position 4 (separated by 35 and $85 \mathrm{~nm}$, respectively) the amplitude of the $G$ band decreases with respect to the $G^{\prime}$ band and the ratio $G / G^{\prime}$ reduces to $\approx 0.7$. Simultaneously, the shape of the $G^{\prime}$ band changes and its center frequency is shifted to $2610 \mathrm{~cm}^{-1}$ whereas the $G$ band remains at $1596 \mathrm{~cm}^{-1}$. For 1 and 2, the $G^{\prime}$ band has a Lorentzian shape while for 3 and 4 two Lorentzian peaks are needed to model its shape. Raman spectra recorded beyond point 4 in Fig. 4(a) remain unchanged.

Variations in the Raman spectrum reflect changes in the molecular structure which can have several origins such as external stress, due to the catalyst particles, or local defects in the tube structure. The observed spectral variations between 1 and 3 can also be explained by a change of the tube structure, described by $(n, m) \rightarrow$ $\left(n^{\prime}, m^{\prime}\right)$, which modifies the electronic state energies of 

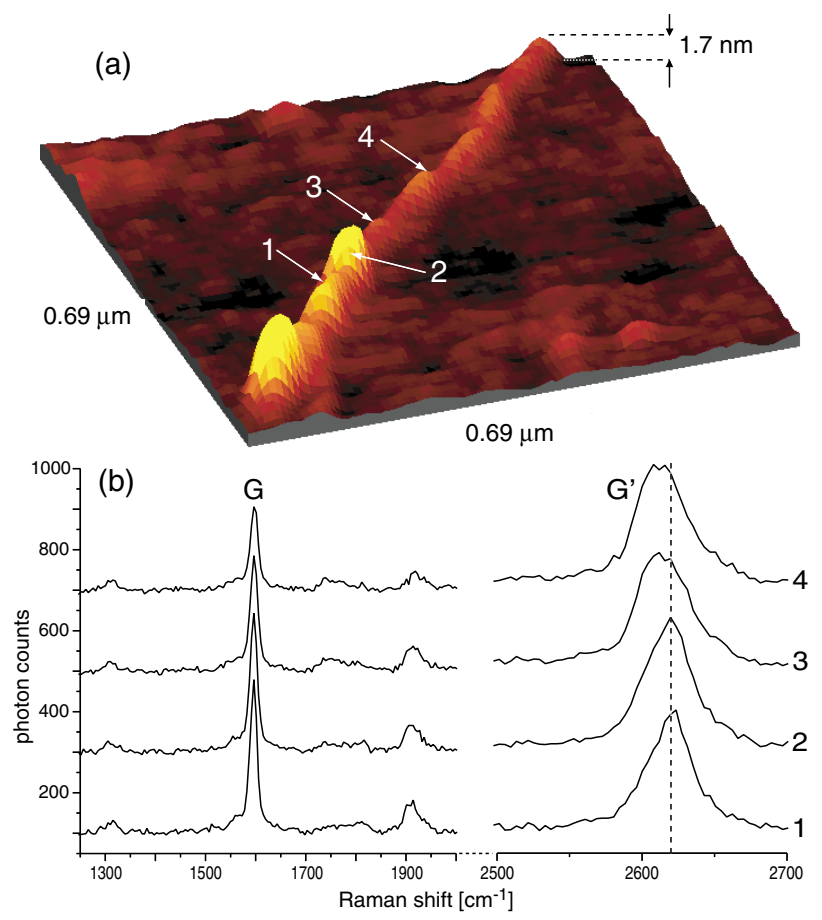

FIG. 4 (color online). (a) Three-dimensional topographic image of a SWNT on glass grown by arc discharge. The three bumps are presumably enclosed $\mathrm{Ni} / \mathrm{Y}$ catalyst particles and indicate the initial point of growth. (b) Near-field Raman spectra detected at the marked positions 1 to 4 in (a). The distance between positions 2 and 3 is $35 \mathrm{~nm}$. The spectra are offset for clarity.

the tube and therefore the resonance enhancement of the Raman bands at a fixed excitation energy. A decrease of the resonance enhancement between positions 2 and 3 would lead to a lower $G$ band amplitude and could cause a splitting of the $G^{\prime}$ band into a double peak [20].

Recent experiments have shown that huge surface enhancement of Raman signals can be accompanied by fluctuating white-light emission [3,23]. According to Michaels et al. [23], huge SERS enhancement and strong white-light emission requires chemisorption of the Raman scatterer to the metal surface. Since we maintain a well-defined separation $(\approx 1 \mathrm{~nm})$ between tip and sample in our experiments, we do not expect a significant white-light contribution. Moreover, white-light emission is observed for Raman enhancement factors more than 10 orders of magnitude stronger than the enhancements determined in our experiments. Indeed, our near-field Raman spectra show no indication for a significant broadband background [see Fig. 4(b)]. To exclude any contributions to the image contrast arising from background signals, we recorded the full spectrum for each pixel and integrated the intensity of the $G^{\prime}$ band and the $G$ band while subtracting the background. The resulting images were virtually identical to those shown in Fig. 2(a). We also note that by comparing the near-field and far-field
Raman spectra of SWNTs, we find, within the noise limit, no indication for a gradient-field Raman effect discussed by Ayars et al. [24].

We have demonstrated a method for nanoscale Raman spectroscopy with sub $30 \mathrm{~nm}$ spatial resolution. This method extends our abilities to characterize weakly fluorescent structures on the nanoscale. Near-field Raman spectroscopy combined with simultaneous AFM measurements appears to be extremely useful for investigations of carbon nanotubes. The method holds promise for the experimental investigation of special structural nanotube features, such as kinks, intertube junctions, or even tubes loaded with $\mathrm{C}_{60}$ molecules.

The authors wish to thank M. Beversluis and A. Bouhelier for stimulating discussions. Assistance on the CVD of SWNTs by Dr. T. Rueckes and Dr. B. Segal of Nantero (Woburn, MA) is gratefully acknowledged. This research was supported by the NSF through Grant No. BES-0086368.

[1] E. J. Sánchez, L. Novotny, and X. S. Xie, Phys. Rev. Lett. 82, 4014 (1999).

[2] K. Kneipp et al., Phys. Rev. Lett. 78, 1667 (1997).

[3] S. Nie and S. R. Emory, Science 275, 1102 (1997).

[4] J. Wessel, J. Opt. Soc. Am. B 2, 1538 (1985).

[5] C. L. Jahncke, H. D. Hallen, and M. A. Paesler, J. Raman Spectrosc. 27, 579 (1996).

[6] S. M. Stöckle et al., Chem. Phys. Lett. 318, 131 (2000).

[7] N. Hayazawa et al., Chem. Phys. Lett. 335, 369 (2001).

[8] L. T. Nieman, G. M. Krampert, and R. E. Martinez, Rev. Sci. Instrum. 72, 1691 (2001).

[9] N. Hayazawa et al., J. Chem. Phys. 117, 1296 (2002).

[10] A. Jorio et al., Phys. Rev. Lett. 86, 1118 (2001).

[11] Z. Yu and L. Brus, J. Phys. Chem. B 105, 1123 (2001).

[12] G. S. Duesberg et al., Phys. Rev. Lett. 85, 5436 (2000).

[13] J. Maultzsch, S. Reich, and C. Thomsen, Phys. Rev. B 65, 233402 (2002).

[14] K. Kneipp et al., Phys. Rev. Lett. 84, 3470 (2000).

[15] K. Karrai and R. D. Grober, Appl. Phys. Lett. 66, 1842 (1995).

[16] M. J. Vasile, C. Biddick, and H. Huggins, Appl. Phys. Lett. 64, 575 (1994).

[17] L. Novotny, E. J. Sánchez, and X.S. Xie, Ultramicroscopy 71, 21 (1998).

[18] B. Sick, B. Hecht, and L. Novotny, Phys. Rev. Lett. 85, 4482 (2000).

[19] J. Hafner, C. L. Cheung, and C. M. Lieber, J. Am. Chem. Soc. 121, 9750 (1999).

[20] A. G. Souza Filho et al., Phys. Rev. B 65, 085417 (2002).

[21] A. Jorio et al., Phys. Rev. Lett. 85, 2617 (2000).

[22] C. Journet et al., Nature (London) 388, 756 (1997).

[23] A. M. Michaels, M. Nirmal, and L. E. Brus, J. Am. Chem. Soc. 121, 9932 (1999).

[24] E. J. Ayars, H. D. Hallen, and C. L. Jahncke, Phys. Rev. Lett. 85, 4180 (2000). 